\title{
Antioxidant content and activity in different parts of pomelo [Citrus grandis (L.) Osbeck] by-products
}

\begin{abstract}
This study aimed to investigate the total antioxidant content and activity in different parts of pomelo by-products, namely flavedo, albedo and segment membrane. Total phenolic content (TPC) was determined using Folin-Ciocalteu method while total flavonoid content (TFC) was determined using aluminium chloride colorimetric method. The total antioxidant activity was determined by performing DPPH free radical scavenging assay and ferric ion reducing antioxidant power (FRAP) assay. Albedo possessed the highest TPC and TFC while flavedo demonstrated the highest antioxidant activity in both assays as compared to other tissues. Pearson correlation tests revealed that there were no correlations between TPC and EC50 as well as FRAP value. There was also a lack of relationship between TFC and antioxidant activity as determined in DPPH and FRAP assays. The pomelo by-products of 'Ledang' possessed higher antioxidant properties than previous studies and therefore can be considered as a new source of natural antioxidants.
\end{abstract}

Keyword: 'Ledang'; Flavedo; Albedo; Segment membrane; Phenolics; Flavonoids 